\title{
Critical Point Theory and the Number of Solutions of a Nonlinear Dirichlet Problem $(*)$.
}

\author{
Alfonso Castro (Mexico D. F., Mexico) - A. C. Lazer (Cincinnati, Ohio)
}

\section{Introduction and Summary.}

The motivation for this paper stems from the following result:

THEorem $D$. - Let $D$ be a bounded domain in $R^{n}$ whose boundary $\partial D$ is of elass $C^{2+\alpha}$ for some $\alpha \in(0,1)$. Let $\Delta$ denote the Laplacian and

$$
0<\lambda_{1}<\lambda_{2} \leqslant \lambda_{3} \leqslant \ldots \leqslant \lambda_{n} \leqslant \ldots
$$

the sequence of eigenvalues of the boundary value problem

$$
\begin{aligned}
& (\Delta u)(x)+\lambda u(x)=0 \quad x \in D \\
& u(x)=0 \quad x \in \partial D,
\end{aligned}
$$

with each $\lambda_{n}$ occurring in the sequence as often as its multiplicity. If $g \in C^{1}(R, R)$, and there exist constants $\gamma$ and $\gamma^{\prime}$ and an integer $N$ such that

$$
\lambda_{N}<\gamma \leqslant g^{\prime}(t) \leqslant \gamma^{\prime}<\lambda_{N+1}
$$

for all $t \in R$, then for any $p \in C^{\alpha}(\bar{D})$ there exists a unique solution of the boundary value problem

$$
\begin{aligned}
A u(x)+g(u(x)) & =p(x) & & x \in D \\
u(x) & =0 & & x \in \partial D .
\end{aligned}
$$

This result was essentially given by C. L. DoLPH in [11]. Although the result is not explicit in [11], it follows immediately from results concerning nonlinear integral equations of the Hammerstein type via use of the Green's function for the boundary value problem $(\Delta u)(x)=f(x), x \in D ; u(x)=0, x \in \partial D$.

For different derivations which depend on implicit function theoretic arguments we refer the reader to the papers [9] and [16]. Generalizations, which give conditions for existence only, can be found in [13] and [14].

(*) Entrata in Redazione 18 settembre 1977.

8-Annali di Matematica 
Aurbrosemtr and Propi studied the boundary value problem $(P)$ under the assumption that the range of $g^{\prime}$ contains an eigenvalue. Specifically, in [2], they showed that if $g(0)=0, g^{\prime \prime}(t)>0$ for all $t$, and $\lim _{t \rightarrow \pm \infty} g^{\prime}(t)=g^{\prime}( \pm \infty)$ with

$$
0<g^{\prime}(-\infty)<\lambda_{1}<g^{\prime}(\infty)<\lambda_{2},
$$

then $(P)$ has either zero, one, or two solutions. More precisely, they showed that in the Banach space $C^{\alpha}(\bar{D})$ there exists a $C^{2}$ closed manifold $M$ whose complement consists of two components $U_{0}$ and $U_{2}$ such that $(P)$ has no solution for $p \in U_{0},(P)$ has two solutions for $p \in U_{2}$, and $(P)$ has one solution for $p \in M$.

In this paper we also consider the boundary value problem under the assumption that the range of $g^{\prime}$ contains an eigenvalue. We shall show that slight alterations of the conditions of Theorem $D$ imply nonuniqueness of the solutions of $(P)$ for suitably restricted $p(x)$. With stronger assumptions on $g$ we can give the exact number of solutions. Specifically, we will prove the following results:

Throrem $A$. - Let $D$ and $p$ satisfy the same smoothness contitions as in Theorem $D$. Assume $g(0)=0, g \in C^{1}(-\infty, \infty)$, and $g^{\prime}$ is bounded. If there exist an integer $N$ and numbers $\gamma$ and $\gamma^{\prime}$ such that $\lambda_{N}<\gamma<\gamma^{\prime}<\lambda_{N+1}$ with $g^{\prime}(t) \leqslant \gamma^{\prime}$ for all $t \in(-\infty, \infty)$ and

$$
-\infty<\inf _{t \in R}\left[\int_{0}^{t} g(s) d s-\frac{\gamma t^{2}}{2}\right],
$$

and if

$$
g^{\prime}(0)<\lambda_{N},
$$

then the homogeneous problem

$$
\begin{aligned}
(\Delta u)(x)+g(u(x)) & =0 & & x \in D \\
u(x) & =0 & & x \in \partial D
\end{aligned}
$$

has at least two solutions; in particular, there exists a nontrivial solution of $\left(P_{0}\right)$. If, in addition to $(* *)$, we assume that

$$
g^{\prime}(0) \neq \lambda_{j} \quad \text { for all } j,
$$

then, if the $L^{2}(D)$ norm of $p(x)$ is sufficiently small, the nonhomogeneous problem $(P)$ has at least three solutions.

THEOREM $B$. - Let $p$ and $D$ satisfy the same smoothness conditions as in Theorem $D$. Assume that $g(0)=0, g \in O^{2}(R, R)$, and that $\operatorname{tg}^{\prime \prime}(t)>0$ almost everywhere. If $\lim _{t \rightarrow \infty} g^{\prime}(t)$ $=g^{\prime}(\infty)$ and $\lim _{i \rightarrow \infty} g^{\prime}(t)=g^{\prime}(-\infty)$ are finite and there exists an integer $N$ such that

(a) $\lambda_{N-1}<g^{\prime}(0)<\lambda_{N}$, 
(b) $\lambda_{N}<g^{\prime}(\infty)<\lambda_{N+1}$,

and

(c) $\lambda_{N}<g^{\prime}(-\infty)<\lambda_{N+1}$,

then there exists a number $r>0$ such that problem $(P)$ has exactly three solutions provided that the $L^{2}(D)$ norm of $p$ is smaller than $r$. We emphasize that it is not necessary that $g^{\prime}(\infty)=g^{\prime}(-\infty)$.

In case the function $g$ is odd we have the following substantial improvement of the first part of Theorem $A$ :

Theorem $O .-$ If the hypotheses of Theorem $A$ are satisfied, if $g$ is odd, and if $K \leqslant N$ is the integer such that

$$
\lambda_{k-1} \leqslant g^{\prime}(0)<\lambda_{k} \leqslant \lambda_{N}
$$

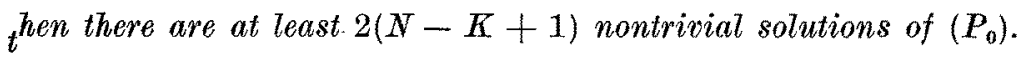

In Theorem $A$, the condition (*) will be satisfied if $\lim _{t} \inf g^{\prime}(t) \geqslant \gamma$. However, $g^{\prime}(t)$ can be less than $\gamma$ for arbitrarily large values of $t^{2}$ and still satisfy condition $(*)$.

Our method of proving all three theorems consists of reducing a certain infinite dimensional problem to a finite dimensional problem and then applying finite dimensional critical point theory. Our main tool from critical point theory, which is Theorem 1 of the next section, appears to be new and we hope that it will have other applications. To prove Theorem $C$ we make use of a result due to CLARK [8] concerning Lusternik-Schnirelman theory.

Although the idea of reducing problems such as $\boldsymbol{P}$ to finite dimensional problems has now become standard (see for example [3] or [6]), our method of reduction is novel in the sense that it involves a variational principle. The abstract methods developed in this paper can obviously be used to treat more general elliptic boundary value problems and nonlinear integral equations of the Hammerstein type-we have considered the simple problem $(P)$ for elarity of exposition.

We mention one open problem related to Theorem $O$ that we have not been able to resolve using our methods. In this case $n=1$ we can show that, if the conditions of Theorem $A$ hold, then the assertion of Theorem $C$ is true without the condition that $g$ be odd. We suspect that this also holds for $n>1$.

\section{1. - Finite dimensional critical point theory.}

The main results of this section will be used only in the proof of the second assertion of Theorem $A$ and in Theorem $B$.

Let $f \in C^{1}\left(R^{n}, R\right)$. If $c$ is a real number we let

$$
f^{\circ}=\left\{x \in R^{n} \mid f(x) \leqslant c\right\},
$$


and

$$
K_{c}=\left\{x \in R^{n} \mid f(x)=o, \nabla f(x)=0\right\}
$$

We say that $f$ satisfies the Palais-Smale condtion, or $(P-S)$ if, whenever $\left\{x_{n}\right\}_{1}^{\infty}$ is a sequence such that $\left\{f\left(x_{n}\right)\right\}_{1}^{\infty}$ is bounded and $\nabla f\left(x_{n}\right) \rightarrow 0$ as $n \rightarrow \infty$, then some subsequence of $\left\{x_{n}\right\}_{1}^{\infty}$ converges.

The following "deformation lemma" is a very special case of a known result due to CLARK [8].

Lemava 1. - If $f \in C^{\mathrm{I}}\left(R^{n}, R\right)$ satisfies condition $(P-S)$, and if for some number $e$, $K_{c}=\Phi$, then there exists a number $\varepsilon>0$ and a continuous function $F: R^{n} \times[0,1] \rightarrow R^{n}$ such that:

(a) $F\left(f^{c+\varepsilon}, 1\right) \subseteq f^{c-\varepsilon}$,

(b) $f(F(x, t)) \leqslant f(x)$ for all $x \in R^{n}, t \in[0,1]$,

(c) $F(x, 0)=x, x \in R^{n}$.

In what follows we let $H_{m}(A)$ denote the $m$-th singular homology group over the integers of a topological space $A ; H_{m}^{\#}(A)$ will denote the corresponding augmented homology group. We let $C_{m}(A)$ denote the group of singular chains on $A$; and if $z \in C_{m}(A)$ is $m$-cycle, with respect to the augmented boundary operator, then [ $\left.z\right]$ $\in H_{m}^{H}(A)$ will denote the augmented homology class of $z$. If $w \in O_{m}(A)$, then $|w| \subset A$ will denote the support of $w$. Finally, if $g: A \rightarrow B$ is continuous, then $C_{m}(g): C_{m}(A) \rightarrow$ $\rightarrow C_{m}(B)$ will denote the chain map induced by $g$, and $H_{m}^{*}(g): H_{m}^{\#}(A) \rightarrow H_{m}^{\#}(B)$ will denote the corresponding augmented homology functor. For further explanation of these terms see [12] or [22].

THEOREM 1. - Let $f \in C^{1}\left(R^{n}, R\right)$ satisfy the $(P-S)$ eondition. If for some number $a$ and some integer $m \geqslant 0, H_{m}^{*}\left(f^{a}\right) \neq\{0\}$, then there exists $\bar{x} \in R^{n}$ such that $f(\bar{x}) \geqslant a$ and $\nabla f(\bar{x})=0$

Proof. - We define a collection $\Sigma$ of compact subsets of $R^{n}$ as follows: Let $z$ be any $m$-cycle on $f^{a}$, with respect to the augmented boundary operator, such that $[z] \neq 0$ in $H_{m}^{*}\left(f^{a}\right)$. Clearly, $z$ is also an (augmented) $m$-cycle on $R^{n}$. Therefore, since $H_{m}^{H}\left(R^{n}\right)=\{0\}$, there exists $w \in O_{m+1}\left(R^{n}\right)$ such that $z=\partial_{m+1} w$, where $\partial_{m+1}: O_{m+1}\left(R^{n}\right) \rightarrow$ $\rightarrow C_{m}\left(R^{n}\right)$ is the $m+1$-dimensional boundary operator. We let $\Sigma$ denote the collection of all supports $|w|$, where $w$ is a singular $(m+1)$-chain on $R^{n}$ that arises in the foregoing manner. The hypothesis of the theorem implies that $\Sigma \neq \Phi$. We claim that

$$
S \cap\{x \mid f(x)>a\} \neq \Phi \text { if } S \in \Sigma .
$$


Indeed, suppose $S=|w|$ where $\partial_{m+1} w=z \in C_{m}\left(f^{a}\right)$ and $[z] \neq 0$ in $H_{m}^{\#}\left(f^{a}\right)$. If (1) did not hold, then $w \in C_{m+1}\left(f^{\alpha}\right)$ and hence $[z]=0$ in $H_{m}^{\#}\left(f^{a}\right)$, which is a contradiction. Consequently,

$$
c=\inf _{S \in \Sigma} \max _{x \in S} f(x) \geqslant a
$$

It follows that the theorem will be proved if we can show that $K_{c} \neq \Phi$. Let us assume that $K_{c}=\Phi$. By Clark's deformation lemma, there exists $F: R^{n} \times[0,1] \rightarrow R^{n}$ such that assertions $(a),(b)$ and $(c)$ of Lemma 1 hold. If $f(x) \leqslant c$, then according to condition $(b), f(F(x, t)) \leqslant \dot{f}(x) \leqslant c$ for $t \in[0,1]$. Hence

$$
F\left(f^{c} \times[0,1]\right) \subset f^{c} .
$$

Let $\bar{F}$ denote the restriction of $F$ to $f^{c} \times[0,1]$ and let $\bar{F}_{k}: f^{c} \rightarrow f^{c}, k=0,1$ be defined by $\bar{F}_{0}(x)=\bar{F}(x, 0)$ and $\bar{F}_{1}(x)=\bar{F}(x, 1)$. Considering the corresponding homology functors, $H_{m}^{\sharp t}\left(\bar{F}_{k}\right): H_{m}^{*}\left(f_{c}\right) \rightarrow H_{m}^{\#}\left(f_{c}\right)$, we infer from (3) and the homotopy invarianee theorem of homology theory (see $\left[12\right.$, p. 45] or $\left[22\right.$, p. 200]) that $H_{m}^{\#}\left(\bar{F}_{0}\right)=H_{m}^{\#}\left(\bar{F}_{1}\right)$. But, from condition $(c)$ of Lemma $1, \bar{F}_{0}(x)=x$ for $x \in f^{c}$, and hence

$$
H_{m}^{H}\left(\bar{F}_{1}\right)=I d .
$$

If $\varepsilon>0$ is as in condition (a) of Lemma 1, then by the definition of $c$ in (2), there exists $S \in \Sigma$ such that $S \subseteq f^{c+s}$. Consequently,

$$
S_{i}=F(S, 1) \subset f^{c-s} \text {. }
$$

Suppose $S=|w|$ with $\partial_{m+1} w=z \in O_{m}\left(f^{a}\right)$, and $[z] \neq 0$ in $H_{m}^{\#}\left(f^{a}\right)$. Let $F(, 1)(x)=$ $=F(x, 1)$ if $x \in R^{n}$. From (4), we have

$$
\left[O_{m}(F(, 1)) z\right]=\left[C_{m}\left(\bar{F}_{1}\right) z\right]=H_{m}^{\#}\left(\bar{F}_{1}\right)([z])=[z] \neq 0 \quad \text { in } H_{m}^{\#}\left(f^{c}\right)
$$

Thus, since

$$
\partial_{m+1} C_{m+1}(F(, 1)) w=C_{m}(F(, 1)) \partial_{m+1} w=O_{m}(F(, 1)) z
$$

it follows that $\left|O_{m+1}(F(, 1)) w\right| \in \Sigma$. Clearly,

$$
\left|O_{m+1}(F(, 1)) w\right|=F(|w|, 1)=F(S, 1)=S_{1},
$$

which proves that $\oiint_{1} \in \Sigma$. However, by $(5) \max _{x \in S_{1}} f(x) \leqslant c-\varepsilon$ wich contradicts the definitions of $c$ given in (2). This contradiction proves that $K_{c} \neq \Phi$ and by earlier remarks it also proves the theorem. 
In the following, if $v: R^{n} \rightarrow R^{n}$ is continuous and $\bar{x}$ is an isolated zero of $v$, then $i(v, \bar{x})$ will denote the index of $v$ at $\bar{x}$ (see $[17$, p. 32$]$ ).

THEOREM 2. - Let $f \in C^{2}\left(R^{n}, R\right)$. If $f(x) \rightarrow \infty$ as $\|x\| \rightarrow \infty$ and the set of solutions of $\nabla f(x)=0$ is a finite set $\left\{x_{0}, x_{1}, x_{2}, \ldots, x_{k}\right\}$, then

$$
\sum_{j=0}^{k} i\left(\nabla f, x_{j}\right)=1
$$

Proof. - Clearly, the growth condition on $f$ implies that $f$ satisfies $(P-S)$. If the number a is chosen so large $f\left(x_{j}\right)<a$ for $j=0,1, \ldots, k$ then, by Theorem 1 , we infer that $H_{m}^{H}\left(f^{a}\right)=\{0\}$ for all $m \geqslant 0$. Hence, $H_{m}\left(f^{a}\right)=\{0\}$ for $m \geqslant 1$, and $H_{0}\left(f^{a}\right)$ is infinite cyclic. Since a is a regular value for $f, f^{a}$ is a $C^{1} n$-manifold with boundary and the $\sigma^{1}$ vector field $\nabla f$ points outward at each boundary point. Since $x_{j} \in f^{a}$, $j=0, \ldots, k$, the Poincaré-Hopf theorem $([17$, p. 35]) implies that

$$
\sum_{j=0}^{k} i\left(\nabla f, x_{j}\right)=\sum_{j=0}^{n}(-1)^{i} \operatorname{rank}\left(H_{j}\left(f^{a}\right)\right)=1
$$

and the theorem is proved.

If $\nabla f(\bar{x})=\mathbf{0}$, we say that $\bar{x}$ is a nondegenerate critical point of $f$ if the Hessian matrix of $f$ at $\bar{x}$ is nonsingular.

THEOREM 3. - Let $f \in C^{2}\left(R^{n}, R\right)$. If $f(x) \rightarrow \infty$ as $\|x\| \rightarrow \infty$; if $\min _{x \in R^{n}} f(x)=f\left(x_{0}\right)$ and if there exists a nondegenerate critical point $x_{1}$ of $f$ such that $x_{1} \neq x_{0}$, then $f$ has at least three distinct oritical points.

Proor. - If $x_{0}$ is a point such that $f\left(x_{0}\right)=\min _{x \in R^{n}} f(x)$, then $\nabla f\left(x_{0}\right)=0$, and if $f$ has an isolated minimum at $x_{0}$, then $i\left(\nabla f, x_{0}\right)=1$. A proof of this geometrically evident fact can be found in [20] or [21]. Since $x_{1}$ is a nondegenerate critical point of $f$, it follows that $i\left(\nabla f, x_{1}\right)= \pm 1$ (see $[17$, p. 37]). If the theorem were false the only critical points of $f$ would be $x_{1}$ and an isolated minimum point $x_{0}$. Consequently, the sum of the indices of $\nabla f$ at its zeros would be 0 or 2 , contradicting Theorem 2 . This proves the result.

REMARK. - Using the reasoning of Theorem 3, RABINowitz [21] proved that if $B$ is a bounded domain, with $\nabla f \neq 0$ on the boundary; if the Brouwer degree $d(\nabla f$, $B, 0)=1$; if $f$ has a local minimum at a point $x_{0} \in B$; and if $f$ has a nondegenerate critical point $x_{1} \in B$ with $x_{1} \neq x_{0}$; then $f$ has at least three critical points in $B$. Assuming that $f$ satisfies the hypotheses of Theorem 2, the proof of Theorem 2 shows that if $B=\{x \mid f(x)<a\}$, then $d(\nabla f, B, 0)=1$ provided a is large. 


\section{2. - An abstract theorem on Hilbert space functionals.}

Our next result is a strengthening of a theorem proved in [5] and [16]. A few details will be omitted in the proof.

Let $H$ be a real Hilbert space and $F$ a real valued function defined on $H$ with a second continuous Fréchet derivative. As is customary, we define a $C^{1}$ map $\nabla F$ : $H \rightarrow H$ such that $F^{\prime}(u)(w)=\langle\nabla F(u), w\rangle$ by means of the Riesz-Frechet theorem. The derivative of $\nabla F$ at $u \in H$, which is a self-adjoint operator on $H$, will be denoted by $D^{2} F(u)$.

THEOREM 4. - Let $F \in C^{2}(H, R)$, and suppose the following conditions are satisfied:

(a) $\nabla F(0)=0$ and there exist elosed subspaces $X_{1}$ and $Y_{1}$ of $H$ and a constant $m_{1}>0$ such that

(i) $\quad H=X_{1} \oplus Y_{1}$,

(ii) $\operatorname{dim} X_{1}<\infty$,

(iii) $\left\langle D^{2} F(0) x, x\right\rangle \leqslant 0$, for all $x \in X_{1}$,

(iv) $\left\langle D^{2} F(0) y, y\right\rangle \geqslant m_{1}\|y\|^{2}$ for all $y \in Y_{1}$.

(b) There exist closed subspaces $X$ and $X$ of $H$ and a constant $m>0$ such that

(v) $H=X \oplus Y$,

(vi) $\operatorname{dim} X_{1}<\operatorname{dim} X<\infty$,

(vii) $(F \mid X)(\infty) \rightarrow-\infty$ as $\|x\| \rightarrow \infty$ (where $F \mid X$ is the restriotion of $F$ to $X$ ),

(viii) $\left\langle D^{2} F(u) y, y\right\rangle \geqslant m\|y\|^{2}$ for all $y \in Y$ and all $u \in H$.

ASSERTION. - There exists $u_{0} \in H$ with $u_{0} \neq 0$ such that $\nabla F\left(u_{0}\right)=0$. Moreover,

$$
F\left(u_{0}\right)=\max _{x \in X} \min _{y \in Y} F(x+y) .
$$

If condition (iii) is replaced by

(iii*) $\left\langle D^{2} F(0) x, x\right\rangle<0$ if $x \in X_{1}$ and $x \neq 0$,

there exists $u_{2} \in H$ with $u_{2} \neq 0$ and $u_{2} \neq u_{0}$ such that $\nabla F\left(u_{2}\right)=0$.

Proof. - For fixed $\hat{x} \in X$ define $g: Y \rightarrow R$ by $g(y)=F(\hat{x}+y)$. If $k \in Y$ then

$$
\langle\nabla g(y), k\rangle=\left.\frac{d}{d t} g(y+t k)\right|_{l=0}=\langle\nabla F(\hat{x}+y), k\rangle
$$

and

$$
\left\langle D^{2} g(y) k, k\right\rangle=\left.\frac{d^{2}}{d t^{2}} g(y+t k)\right|_{t=0}=\left\langle D^{2} F(\hat{x}+y) k, k\right\rangle .
$$


Hence, by (viii), for $y \in Y$ and $k \in Y$,

$$
\left\langle D^{2} g(y) k, k\right\rangle \geqslant m\|k\|^{2} .
$$

As is well-known (see, for example [23, p. 79-80]), (6) implies the existence of $\hat{y} \in K$ such that

$$
g(\hat{y})=\min _{y \in Y} g(y) \quad \text { and } \quad \nabla g(\hat{y})=0 .
$$

Since, for some $s \in(0,1)$,

$$
\left\langle\nabla g\left(y_{1}\right)-\nabla g\left(y_{2}\right), y_{1}-y_{2}\right\rangle=\left\langle D^{2} g\left(y_{2}+s\left(y_{1}-y_{2}\right)\right)\left(y_{1}-y_{2}\right), y_{1}-y_{2}\right\rangle \geqslant m\left\|y_{1}-y_{2}\right\|^{2}
$$

(see $[23, p .37]), \nabla g$ can have only one zero on $Y$ so $\hat{y}$ is unique. Setting $\hat{y}=\varphi(\hat{x})$ this defines a map $\varphi: X \rightarrow Y$. Thus, given $x \in X, \varphi(x)$ is the unique member of $Y$ such that

$$
\langle\nabla F(x+\varphi(x)), k\rangle=0 \quad \text { for all } k \in Y
$$

and such that

$$
F(x+\varphi(x))=\min _{y \in Y} F(x+y) .
$$

A simple argument based on the implicit function theorem shows that the condition (viii) implies that $\varphi$ is of class $C^{1}$. See $[16, p$. 597-598] for the details.

We claim that the function $G: X \rightarrow R$, which is defined by $G(x)=F(x+\varphi(x))$, is of class $C^{2}$. This is not immediately apparent since we only know that $\varphi \in C^{1}(Y, X)$. If $h \in X$, we have

$$
\begin{aligned}
\langle\nabla G(x), h\rangle=\left.\frac{d}{d t} G(x+t h)\right|_{l=0}=\left.\frac{d}{d t} F(x+t h+\varphi(x+t h))\right|_{t=0} & = \\
& =\left\langle\nabla F(x+\varphi(x)), h+\varphi^{\prime}(x)(h)\right\rangle .
\end{aligned}
$$

Since $\varphi^{\prime}(x)$ is a linear map from $X$ to $Y$ we see that $k=\varphi^{\prime}(x)(h) \in Y$, so by (7) and the above

$$
\langle\nabla G(x), h\rangle=\langle\nabla F(x+\varphi(x)), h\rangle
$$

Since $F$ is of class $C^{2}, \nabla F$ is of class $C^{1}$; hence, since $\varphi \in C^{1}$, it follows that $\langle\nabla G(x), h\rangle$ is of class $C^{1}$ for all $h \in K$. This implies that $G$ is of class $C^{2}$, and if $h \in X$,

$$
\begin{aligned}
\left\langle D^{2} G(x) h, h\right\rangle=\left.\frac{d}{d t}\langle\nabla G(x+t h), h\rangle\right|_{t=0}= & \left.\frac{d}{d t}\langle\nabla F(x+t h+\varphi(x+t h)), h\rangle\right|_{t=0} \\
& =\left\langle D^{2} F^{\prime}(x+\varphi(x))\left(h+\varphi^{\prime}(x)(h)\right), h\right\rangle .
\end{aligned}
$$


In order to obtain another expression for $\left\langle D^{2} G(x) h, h\right\rangle$ we observe from (7) that if $h \in X$ and $k \in Y$ then $\langle\nabla F(x+t h+\varphi(x+t h)), k\rangle=0$ for all $t$.

Hence,

$$
\begin{aligned}
& \left.\frac{d}{d t}\langle\nabla F(x+t h+\varphi(x+t h)), k\rangle\right|_{t=0}= \\
& =\left\langle D^{2} F(x+\varphi(x))\left(h+\varphi^{\prime}(x)(h)\right), k\right\rangle=0 \quad \text { if } k \in Y .
\end{aligned}
$$

Therefore, by setting $k=\varphi^{\prime}(x)(h) \in Y$, we see from (10) that, if $h \in X$, then

$$
\left\langle D^{2} G(x) h, h\right\rangle=\left\langle D^{2} F(x+\varphi(x))\left(h+\varphi^{\prime}(x)(h)\right), h+\varphi^{\prime}(x)(h)\right\rangle .
$$

To prove the first assertion of Theorem 4, we observe, by setting $y=0$ in (8), that

$$
F(x+\varphi(x)) \leqslant F(x) \quad \text { if } x \in X .
$$

Hence, according to assumption (viii),

$$
G(x)=F(x+\varphi(x)) \rightarrow-\infty \quad \text { as }\|x\| \rightarrow \infty .
$$

Since $\operatorname{dim} X<\infty$, this implies the existence of $x_{0} \in X$ with

$$
G\left(x_{0}\right)=\max _{x \in X} G(x)
$$

Therefore, if $h \in X$ is arbitrary, we infer from (9) that $\left\langle\nabla G\left(x_{0}\right), h\right\rangle=\left\langle\nabla F\left(x_{0}\right.\right.$ $\left.\left.+\varphi\left(x_{0}\right)\right), h\right\rangle=0$. Since $H=X \oplus Y$, if $w \in H, w=h+7$ with $h \in X$ and $k \in Y$, so by (7) and the above $\left\langle\nabla F\left(x_{0}+\varphi\left(x_{0}\right)\right), w\right\rangle=0$. Consequently, if $u_{0}=x_{0}+\varphi\left(x_{0}\right)$ then $\nabla E\left(u_{0}\right)=0$, and from (8) and (14) it follows that

$$
F\left(u_{0}\right)=\max _{\tilde{z} \in X} F(x+\varphi(x))=\max _{x \in X} \min _{y \in Y} F(x+y) .
$$

To complete the proof of the first assertion we must show that $u_{0} \neq 0$. To this end we consider the subspace $W$ of $H$ defined by

$$
W=\left\{w \in H \mid w=h+\varphi^{\prime}\left(x_{0}\right)(h), h \in X\right\}
$$

Since $\varphi^{\prime}\left(x_{0}\right)(h) \in Y$ if $h \in X$ and $X \cap Y=\{0\}$, it follows that $\operatorname{dim} W=\operatorname{dim} X$. If $h \in X$, then by (14),

$$
\left\langle D^{2} G\left(x_{0}\right) h, h\right\rangle=\left.\frac{d^{2}}{d t^{2}} G\left(x_{0}+t h\right)\right|_{t=0} \leqslant 0 .
$$


Hence, using (12), we see that

$$
\left\langle D^{2} F\left(x_{0}+\varphi\left(x_{0}\right)\right)\left(h+\varphi^{\prime}\left(x_{0}\right)(h)\right), h+\varphi^{\prime}\left(x_{0}\right)(h)\right\rangle \leqslant 0
$$

for all $h \in X$, and so

$$
\left\langle D^{2} F\left(u_{0}\right) w, w\right\rangle \leqslant 0 \quad \text { for } w \in W
$$

Let $w_{1}, \ldots, w_{m}$ be a basis for $W$. According to (i) $H=X_{1} \oplus Y_{1}$ so there exist $r_{k} \in X_{1}$ and $s_{k} \in Y_{1}$ for $k=1, \ldots, m$ such that

$$
w_{k}=r_{k}+s_{k}, \quad k=1, \ldots, m .
$$

By (vi), $\operatorname{dim} X_{x}<\operatorname{dim} X=\operatorname{dim} W=m$, so there exist numbers $e_{1}, \ldots, c_{m}$, not all zero, such that $c_{1} r_{1}+\ldots+c_{m} r_{m}=0$; therefore

$$
\hat{w}=\sum_{k=1}^{m} e_{k} w_{k}=\sum_{k=1}^{m} c_{k} s_{k} \in Y_{\mathbf{1}}
$$

and $\hat{w} \neq 0$. Thus by condition (iv), $\left\langle D^{2} F(0) \hat{w}, \hat{w}\right\rangle>0$. Since $\hat{w} \in W$, it follows from (15) that $u_{0} \neq 0$, and the proof of the first part of Theorem 4 is complete.

To prove the second assertion of Theorem 4, we first observe that 0 is a critical point of $G$ distinct from $x_{0}$. Indeed, since $\nabla F(0)=0$, and since according to (6), given $x \in X, \varphi(x)$ is the unique member of $Y$ such that $\langle\nabla F(x+\varphi(x)), k\rangle=0$ for all $k \in Y$, it follows that $\varphi(0)=0$. Therefore, by $(9)$, if $h \in X,\langle\nabla G(0), h\rangle=\langle\nabla F(0$ $+\varphi(0)), h\rangle=\langle\nabla F(0), h\rangle=0$, so $\nabla G(0)=0$. Since, as shown above $u_{0}=x_{0}$ $+\varphi\left(x_{0}\right) \neq 0$, it follows that $x_{0} \neq 0$.

We claim that the condition (iii*) implies that 0 is a nondegenerate critical point. To see this, we first show that the kernel of $D^{2} F(0)$ is trivial. If $D^{2} F^{\prime}(0) u=0$ and $u=r+s$ with $r \in X_{1}$ and $s \in Y_{1}$ then the self-adjointness of $D^{2} F(0)$ implies that

$$
0=\left\langle r-s, D^{2} F(0)(r+s)\right\rangle=\left\langle r, D^{2} F(0) r\right\rangle-\left\langle s, D^{2} F(0) s\right\rangle .
$$

Since conditions (iii*) and (iv) imply that $\left\langle s, D^{2} F(0) s\right\rangle\langle 0$ unless $s=0$, and $\langle r$, $D^{2} F(0) r>>0$ unless $r=0$, it follows that $u=0$. Suppose now that for some $h_{1} \in X$ $D^{2} G(0) h_{1}=0$. It follows from (9) that if $h_{2} \in X$ then

$$
\begin{aligned}
0=\left\langle D^{2} G(0) h_{1}, h_{2}\right\rangle=\left.\frac{d}{d t}\left\langle\nabla Q\left(t h_{1}\right), h_{2}\right\rangle\right|_{t=0} \\
=\left.\frac{d}{d t}\left\langle\nabla F\left(t h_{1}+\varphi\left(t h_{1}\right)\right), h_{2}\right\rangle\right|_{t=0}=\left\langle D^{2} F(0)\left(h_{1}+\varphi^{\prime}(0) h_{1}\right), h_{2}\right\rangle=0 .
\end{aligned}
$$


From (11) we see that if $k \in Y$ then

$$
\left\langle D^{2} F(0)\left(h_{1}+\varphi^{\prime}(0) h_{1}\right), k\right\rangle=\left\langle D^{2} F(0+\varphi(0))\left(h_{1}+\varphi^{\prime}(0) h_{1}\right), k\right\rangle=0 .
$$

Since $H=X \oplus Y$, this and (16) show that $\left\langle D^{2} F^{\prime}(0)\left(h_{1}+\varphi^{\prime}(0) h_{1}\right), u\right\rangle=0$ for all $u \in H$. Hence $h_{1}+\phi^{\prime}(0) h_{1}=0$ so $h_{1}=0$. This proves the claim that the linear map $D^{2} G(0): X \rightarrow X$ is nonsingular.

Consider the function $f: X \rightarrow R$ defined by $f(x)=-G(x)$. By (13) and (14), $f(x) \rightarrow \infty$ as $\|x\| \rightarrow \infty$ and $f\left(x_{0}\right)=\min _{x \in X} f(x)$. As shown above 0 is a nondegenerate critical point of $f$ distinct from $x_{0}$. Therefore, by Theorem 3 of the previous section, there exists a point $x_{2} \in X$, with $x_{2} \neq x_{0}$ and $x_{2} \neq 0$ such that $\nabla f\left(x_{2}\right)=0$. Therefore if $h \in X$, it follows from (9) that $\left\langle\nabla F\left(x_{2}+\varphi\left(x_{2}\right)\right), h\right\rangle=\left\langle\nabla G\left(x_{2}\right), h\right\rangle=0$. Since according to $(7)\left\langle\nabla F\left(x_{2}+\varphi\left(x_{2}\right)\right), k\right\rangle=0$ for all $k \in Y$, we infer that $\nabla F\left(x_{2}+\varphi\left(x_{2}\right)\right)$ $=0$. Finally, since $X \cap Y=\{0\}$, we see that $u_{2}=x_{2}+\varphi\left(x_{2}\right) \neq x_{0}+\varphi\left(x_{0}\right)=u_{0}$, and $u_{2} \neq 0$. This completes the proof of Theorem 4 .

\section{3. - Proof of Theorem $A$.}

We shall first prove Theorems $A$ and $B$ for weak solutions and defer discussion of regularity until the end of the paper.

To derive Theorem $A$ from Theorem 4 we take as our real Hilbert space $H$ the Sobolev space $\stackrel{\circ}{H}_{1}(D)$ which is the completion of the inner product space consisting of real $C^{1}$ functions having support contained in $D$ with inner product

$$
\langle u, v\rangle_{\mathbf{1}}=\int_{D}\langle\nabla u(x), \nabla v(x)\rangle d x .
$$

We let $\langle,\rangle_{0}$ denote the usual $L^{2}(D)$ inner product. If $\left\{\lambda_{m}\right\}_{1}^{\infty}$ is the sequence defined in the introductory section, if for each $m, \varphi_{m}$ satisfies $\left(\Delta \varphi_{m}\right)(x)+\lambda_{m} \varphi_{m}(x)=0$ if $x \in D$ and $\varphi_{m}(x)=0$ for $x \in \partial D$, and if $\left\langle\varphi_{m}, \varphi_{l}\right\rangle_{0}=\delta_{m l}$, then for all $u \in H$

$$
\langle u, u\rangle_{1}=\sum_{m=1}^{\infty} \lambda_{m}\left\langle\varphi_{m}, u\right\rangle_{0}^{2}
$$

and

$$
\langle u, u\rangle_{0}=\sum_{m=1}^{\infty}\left\langle\varphi_{m}, u\right\rangle_{0}^{2}
$$

(see, for example, [9] or [4]). If $N$ is the integer that appers in the statement of Theorem $A$, we let $X$ denote the finite dimensional subspace of $H$ spanned by $\varphi_{1}$, $\varphi_{2}, \ldots, \varphi_{N}$ and $Y=X^{\perp}$. From (17) and (18) it follows that

$$
\langle y, y\rangle_{1} \geqslant \lambda_{N+1}\langle y, y\rangle_{0} \quad \text { if } y \in Y,
$$


and

$$
\langle x, x\rangle_{1} \leqslant \lambda_{N}\langle x, x\rangle_{0} \quad \text { if } x \in X
$$

Let $K \leqslant N$ be the integer such that

$$
\lambda_{R-1} \leqslant g^{\prime}(0)<\lambda_{R} \leqslant \lambda_{N},
$$

where $\lambda_{0}=-\infty$, and let $X_{1}$ denote the span of $\left\{\varphi_{1}, \ldots, \varphi_{K_{-1}}\right\}$ and $Y_{1}=X_{1}^{\perp}$. We define $F: H \rightarrow R$ by

$$
F(u)=\frac{\langle u, u\rangle_{1}}{2}-\int_{D} V(u(x)) d x
$$

where $V(t)=\int_{0}^{t} g(s) d s$. As is shown in [16], the boundedness and continuity of $g^{\prime}$ implies that $F \in C^{2}(H, R)$. Moreover, if $w \in H$

$$
\langle\nabla F(u), w\rangle_{1}=\left.\frac{d}{d t} F(u+t w)\right|_{t=0}=\langle u, w\rangle_{1}-\int_{D} g(u(x)) w(x) d x .
$$

Therefore, weak solutions of the boundary value problem $\left(P_{0}\right)$ coincide with critical points of $F$.

If $u, v$, and $w$ are in $H$, then

$$
\left\langle D^{2} F(u) v, w\right\rangle_{\mathbf{1}}=\left.\frac{d}{d t}\langle\nabla F(u+t v), w\rangle_{1}\right|_{t=0}=\langle v, w\rangle_{\mathbf{1}}-\int_{D} g^{\prime}(u(x)) v(x) w(x) d x .
$$

Thus from (19) and the hypothesis of Theorem $A$, we see that if $y \in Y$,

$$
\left\langle D^{2} F(u) y, y\right\rangle_{1} \geqslant\langle y, y\rangle_{1}-\gamma^{\prime}\langle y, y\rangle_{0} \geqslant m\langle y, y\rangle_{1}, \quad m=1-\frac{\gamma^{\prime}}{\lambda_{N+1}}>0 .
$$

Hence condition (viii) of Theorem 4 is satisfied. If $x \in X$, it follows from condition (*) of Theorem $A,(20)$, and (22) that for some constant $e$

$$
F(x) \leqslant \frac{\langle x, x\rangle_{1}}{2}-\frac{\gamma\langle x, x\rangle_{0}}{2}+c \leqslant \frac{1}{2}\left(1-\frac{\gamma}{\lambda_{N}}\right)\langle x, x\rangle_{1}+c .
$$

Since $\lambda_{N}<\gamma$ we see that condition (vii) of Theorem 4 si satisfied.

From the definition of $X_{1}$ and $Y_{1}$, and (17)-(18), we have $\langle r, r\rangle_{1} \leqslant \lambda_{K_{-1}}\langle r, r\rangle_{0}$ if $r \in X_{1}$ and $\langle s, s\rangle_{1} \geqslant \lambda_{K}\langle s, s\rangle_{0}$ if $s \in Y_{1}$. Consequently, from (21) and (23) we see that, if $r \in X_{1}$, then

$$
\left\langle D^{2} F(0) r, r\right\rangle_{1}=\langle r, r\rangle_{1}-\int_{D} g^{\prime}(0) r(x)^{2} d x \leqslant\langle r, r\rangle_{1}-\lambda_{R-1}\langle r, r\rangle_{0} \leqslant 0
$$


while, if $s \in Y_{1}$, then

$$
\left.\left\langle D^{2} F(0) s, s\right\rangle_{1}=\langle s, s\rangle_{1}-g^{\prime}(0)\langle s, s\rangle_{0}\right\rangle\left(1-\frac{g^{\prime}(0)}{\lambda_{K}}\right)\langle s, s\rangle_{1}=m_{1}\langle s, s\rangle_{1} .
$$

Since $m_{1}>0$, conditions (iii) and (iv) of Theorem 4 are verified. Since the remainder of the conditions (i)-(viii) of Theorem 4 are obviously satisfied, it follows that conditions (*) and (**) of Theorem $A$ imply the existence of at least two solutions of problem $\left(P_{0}\right)$.

Suppose now that condition (***) of Theorem $A$ is satisfied. In this case we see from (23) that

$$
\lambda_{k-1}<g^{\prime}(0)<\lambda_{R}
$$

If $X_{1}$ and $Y_{1}$ are defined as before, then the inequality $\left\langle D^{2} F(0) s, s\right\rangle_{1} \geqslant m_{1}\left\langle s, s_{1}\right\rangle_{1}$ for $s \in Y_{1}$ is still valid and our previous reasoning shows that if $r \in X_{1}$, then

$$
\left\langle D^{2} F(0) r, r\right\rangle_{1}=\langle r, r\rangle_{1}-g^{\prime}(0)\langle r, r\rangle_{0} \leqslant\left[1-\frac{g^{\prime}(0)}{\lambda_{E-1}}\right]\langle r, r\rangle_{1}=-m_{2}\langle r, r\rangle_{1}
$$

where $m_{2}>0$. Since $D^{2} F$ is continuous there exists $\delta_{1}>0$ such that $\mid D^{2} F(u)-$ $-D^{2} F(0) \mid<\min \left(m_{1} / 2, m_{2} / 2\right)$ if $|u|<\delta_{1}$. Hence, by the above

$$
\begin{array}{ll}
\left\langle D^{2} F(u) r, r\right\rangle_{1} \leqslant-\frac{m_{2}}{2}\langle r, r\rangle_{1}, & r \in X_{1}, \\
\left\langle D^{2} F(u) s, s\right\rangle_{1} \geqslant \frac{m_{1}}{2}\langle s, s\rangle_{1}, & s \in Y_{1},
\end{array}
$$

if $|u|<\delta_{1}$.

From (23), if $v, w \in H,\left\langle D^{2} F(0) v, w\right\rangle_{1}=\langle v, w\rangle_{1}-g^{\prime}(0)\langle v, w\rangle_{0}$; hence, $D^{2} F(0) v=$ $=v-g^{\prime}(0) T v$, where $T$ is the linear operator on $H$ defined by $\langle T v, w\rangle_{1}=\langle v, w\rangle_{0}$. Since the injection from $\stackrel{\Omega}{H}_{1}(D)$ into $L^{2}(D)$ is compact, $T$ is a compact operator on $H$. If $u \in$ kernel $D^{2} F(0)$, then $u$ is a weak solution of $\Delta u+g^{\prime}(0) u=0$ in $D, u=0$ on $\partial D$. Since, by standard regularity theory, $u$ is a classical solution, we see from (25) that $u=0$. Hence, by the Fredholm alternative, the continuous linear map $D^{2} F(0)$ $D^{2} F(0): H \rightarrow H$ is one-to-one and onto. Therefore, since $\nabla F(0)=0$, it follows from the inverse function theorem (see, for example [15]) that there exists an open set $U$ in $H$ containing 0 such that the restriction of $\nabla F$ to $U$ is one-to-one, $\nabla F(U)$ is an open set containing 0 , and $\nabla F$ restrieted to $U$ has a $C^{1}$ inverse. Without loss of generality we may assume that $|u|_{1}<\delta_{1}$ for all $u \in U$.

Suppose that $r>0$ is such that $|v|_{1}<r$ implies that $v \in \nabla F(U)$. We claim that if $p \in L^{2}(D)$ and $|p|_{0}<\sqrt{\lambda_{1}} r$, then there exists a unique weak solution $\varphi$ of the problem $\Delta u+g(u)=p(x), u=0$ on $\partial D$ such that $|\varphi|_{1}<\delta_{1}$. To see this we note that $-<p, w\rangle_{0}, w \in \AA_{1}(D)$, represents a continuous linear functional on $H$ so according 
to the Riesz representation theorem there exists $v \in \stackrel{ }{H}_{1}(D)$ such that $-\langle p, w\rangle_{0}=$ $=\langle v, w\rangle_{1}$. By the Schwarz inequality, (17), and (18)

$$
|v|_{1}^{2} \leqslant|p|_{0}|v|_{0} \leqslant|p|_{0} \frac{1}{\sqrt{\lambda_{1}}}|v|_{1}
$$

hence $|v|_{1}<r$. Therefore there exists $\varphi$ with $|\varphi|_{1}<\delta_{1}$ such that $\nabla F(\varphi)=v$. Consequently, for $w \in \stackrel{\cap}{H}_{1}(D),\left\langle\nabla F^{\prime}(\varphi), w\right\rangle_{1}=\langle v, w\rangle_{1}=-\langle p, w\rangle_{0}$ or, by $(22)$

$$
\int_{D}(\langle\nabla \varphi, \nabla w\rangle-g(\varphi) w+p w) d x=0 .
$$

This shows that $\varphi$ is a weak solution of $\Delta u+g(u)=p$.

We now fix $p$ and $\varphi$ and define $F_{1}: H \rightarrow R$ by

$$
F_{1}(u)=\int_{D}\left(\frac{\langle\nabla(u+\varphi), \nabla(u+\varphi)\rangle}{2}-\nabla(u+\varphi)+p[u+\varphi]\right) d x .
$$

We will show that $F_{1}$ satisfies the conditions of Theorem 4, with (iii) replaced by (iii*). Since for $w \in \stackrel{\circ}{H}_{1}(D)$

$$
\left\langle\nabla F_{1}(u), w\right\rangle_{1}=\left.\frac{d}{d t} F_{1}(u+t w)\right|_{\ell=0}=\int_{D}(\langle\nabla(u+\varphi), \nabla w\rangle-g(u+\varphi) w+p w) d x
$$

we see that $\nabla F_{1}(0)=0$ and that $\nabla F_{1}(u)=0$ if and only if $u+\varphi$ is a weak solution of $(P)$. Moreover, since

$$
\left\langle D^{2} F_{1}(u) v, w\right\rangle_{1}=\left.\frac{d}{d t}\left\langle\nabla F_{1}(u+t v), w\right\rangle_{1}\right|_{t=0}=\int_{D}\left(\langle\nabla v, \nabla w\rangle-g^{\prime}(u+\varphi) v w\right) d x,
$$

it follows from (23) that $D^{2} F_{1}(u)=D^{2} F(u+\varphi)$. Consequently, if $y \in Y$, we see from (24) that

$$
\left\langle D^{2} F(u) y, y\right\rangle_{1} \geqslant m\langle y, y\rangle_{1}
$$

for all $u \in H$. This shows that condition (viii) of Theorem 4 holds for $F_{1}$. Since $|\varphi|_{1}<\delta_{1}$, we see from (26) and (27) that, if $r \in X_{1}$, then

$$
\left\langle D^{2} F_{1}(0) r, r\right\rangle_{1}=\left\langle D^{2} F(p) r, r\right\rangle_{1} \leqslant-\frac{m_{2}}{2}\langle r, r\rangle_{1}
$$

while if $s \in Y_{1}$,

$$
\left\langle D^{2} F_{1}(0) s, s\right\rangle_{1}=\left\langle D^{2} F(\varphi) s, s\right\rangle_{1} \geqslant \frac{m_{1}}{2}\left\langle s, s_{1}\right\rangle_{1} .
$$

Thus $F_{1}$ satisfies conditions (iii*) and (iv) of Theorem 4. 
To see that $F_{1}$ satisfies condition (vii) of Theorem 4 , let $x \in X$. From (20) and (*) of Theorem $A$, we see that for some constants $e$ and $c^{\prime}$

$$
\begin{aligned}
F_{1}(x) & =\frac{\langle x+\varphi, x+\varphi\rangle_{1}}{2}-\int_{D} V(x(\xi)+\varphi(\xi)) d \xi+\langle p, x\rangle_{0} \\
& \leqslant \frac{1}{2}|x+\varphi|_{1}^{2}-\frac{\gamma}{2}|x+\varphi|_{0}^{2}+c+|p|_{0}|x|_{0} \\
& \leqslant \frac{1}{2}\left[|x|_{0}^{2}-\gamma|x|_{0}^{2}\right]+|\varphi|_{1}|x|_{1}+\left(|p|_{0}+\gamma|\varphi|_{0}\right)|x|_{0}+c^{\prime} \\
& \leqslant \frac{1}{2}\left(1-\frac{\gamma}{\lambda_{N}}\right)|x|_{1}^{2}+|\varphi|_{1}|x|_{1}+\frac{1}{\sqrt{\lambda_{1}}}\left(|p|_{0}+\gamma|\varphi|_{0}\right)|x|_{1}+c^{\prime}
\end{aligned}
$$

Hence $\left(F_{1} \mid X\right)(x) \rightarrow-\infty$ as $|x|_{1} \rightarrow \infty$ and $F_{1}$ satisfies condition (vii). Since the remaining conditions of Theorem 4 obviously hold, it follows from the second assertion of Theorem 4 that there exist $u_{0}$ and $u_{2}$ with $u_{0} \neq u_{2}, u_{0} \neq 0, u_{2} \neq 0$ such that $\nabla F_{1}(\varphi$ $\left.+u_{k}\right)=0, k=0,2$. By the above remarks, $\varphi, \varphi+u_{0}$, and $\varphi+u_{2}$ are distinct weak solutions of problem $(P)$.

\section{4. - Proof of Theorem $B$.}

We shall prove Theorem $B$ via several lemmas. The first, which is stated for future reference, is essentially implicit in the proof of Theorem 4.

Lemia 1. - Let $H$ be a real Hilbert space and let $F \in O^{2}(H, R)$ satisfy conditions (v), (vii), and (viii) of Theorem 4 with $\operatorname{dim} X<\infty$. In order that $\nabla F(u)=0$ it is necessary and sufficient that for some $x \in X, u=x+\varphi(x)$ and $\nabla G(x)=0$ where $\varphi: X \rightarrow Y$ and $G: X \rightarrow R$ are defined by (8) and (13) respectively.

The sufficiency of these conditions follows immediately from (7), (9), and condition ( $\nabla)$. As shown in the proof of Theorem 4, if for a given $\hat{x} \in X$ there exists a $y \in Y$ such that $\langle\nabla F(\hat{x}+y), k\rangle=0$ for all $k \in Y$, then $y=\varphi(\hat{x})$. Therefore, the necessity is elear from (v) and (9).

Assume that the function $g$ satisfies the conditions of Theorem $B$. From the condition $t g^{\prime \prime}(t)>0$, a.e. and conditions $(a),(b)$ and $(c)$ it is clear that $g$ satisfies the hypotheses of the first part of Theorem $A$, where we may choose $\gamma$ and $\gamma^{\prime}$ to be any numbers satisfying

$$
\lambda_{N}<\gamma<\min \left\{g^{\prime}(-\infty), g^{\prime}(\infty)\right\} \leqslant \max \left\{g^{\prime}(-\infty), g^{\prime}(\infty)\right\} \leqslant \gamma^{\prime}<\lambda_{N_{+1}}
$$

Moreover, from $(a), g$ satisfies condition $(* * *)$ of Theorem $A$. Therefore, referring 
to the proof of Theorem $A$ and letting the functions $\left\{\varphi_{m}\right\}_{1}^{\infty}$ have the same meaning as before, we have

LEMMA 2. - Let $g$ satisfy the conditions of Theorem $B$. If $H=\stackrel{\circ}{H}_{1}(D)$ and $F$ is defined as in (22) then $F$ satisties conditions (i), (ii), (iii*), (iv)-(viii) of Theorem 4 where

$$
X=\operatorname{span}\left\{\varphi_{1}, \ldots, \varphi_{N}\right\}, \quad Y=X^{\perp}
$$

and

$$
X_{1}=\operatorname{span}\left\{\varphi_{1}, \ldots, \varphi_{N-1}\right\}, \quad Y_{1}=X_{1}^{\perp} .
$$

LeMcr 3. - Assume that the hypotheses of Theorem $B$ hold. Let $F$ be defined as in Lemma 2 and suppose that $\nabla F\left(u_{0}\right)=0$ so, by Lemma $1, u_{0}=x_{0}+\varphi\left(x_{0}\right)$ with $x_{0} \in X$ and $\nabla G\left(x_{0}\right)=0$. If $u_{0} \neq 0$ then $\operatorname{sgn} \operatorname{det} D^{2} G\left(x_{0}\right)=(-1)^{N}$ and $\operatorname{sgn} \operatorname{det} D^{2} G(0)=(-1)^{N-1}$.

Proof. - Suppose $\nabla F\left(u_{0}\right)=0$ and $u_{0} \neq 0$. As shown in the proof of Theorem $A$,

$$
\Delta u_{0}+g\left(u_{0}\right)=0 \quad \text { in } D, \quad u_{0} \mid \partial D=0
$$

in the weak sense. Since $g(0)=0$ we may rewrite (29) in the form

$$
\Delta u_{0}+q(x) u_{0}=0 \quad \text { in } D, \quad u_{0} \mid \partial D=0
$$

where

$$
q(x)=\int_{0}^{1} g^{\prime}\left(s u_{0}(x)\right) d s
$$

In the last section it will be shown that, as a result of standard regularity theory, $u_{0}$ is actually a classical solution of $(30)$. Assuming that this is true for the time being, the simple form of $(30)$ and known results on unique continuation $([4$, p. 160163], [18, p. 59-61]) imply that $u_{0}$ cannot vanish identically on an open subset of $D$.

We consider the two eigenvalue problems

$$
\Delta w+\alpha q(x) w=0 \quad \text { in } D, \quad w \mid \partial D=0
$$

and

$$
\Delta w+\beta g^{\prime}\left(u_{0}(x)\right) w=0 \quad \text { in } D, \quad w \mid \partial D=0 .
$$

Let

$$
\alpha_{1}<\alpha_{2} \leqslant \ldots \leqslant \alpha_{k} \leqslant \alpha_{k+1} \leqslant \cdots
$$

and

$$
\beta_{1}<\beta_{2} \leqslant \ldots \leqslant \beta_{k} \leqslant \beta_{k+1} \leqslant \cdots
$$


denote the eigenvalues of the problems (32) and (33) respectively, with each eigenvalue occuring as often as the number of independent solutions associated with it. We claim that

$$
\beta_{N}<1<\beta_{N+1}
$$

where $N$ is the integer which occurs in the statement of Theorem $B$. To see this, let $\left\{\psi_{k}\right\}_{k=1}^{\infty}$ and $\left\{\theta_{k}\right\}_{k=1}^{\infty}$ be sequences of functions in $\stackrel{\circ}{H}_{1}(D)$ such that

$$
\Delta \psi_{k}+\alpha_{k} q(x) \psi_{k}=0, \quad \Delta \theta_{k}+\beta_{k} g^{\prime}\left(u_{0}(x)\right) \theta_{k}=0
$$

for all $k$, and such that

$$
\int_{D} q(x) \psi_{k}(x) \psi_{j}(x) d x=\delta_{k j}, \quad \int_{D} g^{\prime}\left(u_{0}(x)\right) \theta_{k}(x) \theta_{j}(x) d x=\delta_{k j} .
$$

For fixed $k$, choose numbers $c_{1}, \ldots, c_{k}$ not all zero such that if $v=\sum_{j=1}^{k} c_{j} \psi_{j}$ then

$$
\int_{D} g^{\prime}\left(u_{0}(x)\right) v(x) \theta_{j}(x) d x=0, \quad j=1, \ldots, k-1 .
$$

According to the Rayleigh quotient characterization of the eigenvalues of (33) ([9]) we have

$$
\beta_{k} \leqslant \int_{D}\langle\nabla v, \nabla v\rangle d x / \int_{D} g^{\prime}\left(u_{0}\right) v^{2} d x
$$

Since $t g^{\prime \prime}(t)>0$ for almost all $t \in(-\infty, \infty)$, we see that if $x \in D$ and $u_{0}(x) \neq 0$, then

$$
q(x)=\int_{0}^{1} g^{\prime}\left(s u_{0}(x)\right) d s<g^{\prime}\left(u_{0}(x)\right) .
$$

Therefore, since $u_{0}(x)$ cannot vanish identically on any open subset of $D$, we see from (35) that

and hence

$$
\beta_{k}<\int_{D}\langle\nabla v, \nabla v\rangle d x / \int_{D} q(x) v^{2} d x=\sum_{i=1}^{k} \alpha_{j} e_{i}^{2} / \sum_{j=1}^{k} c_{j}^{2},
$$

$$
\beta_{k}<\alpha_{k}, \quad k=1,2, \ldots
$$

We now consider the two eigenvalue problems

$$
\Delta w+\gamma \lambda_{N+1} w=0 \quad \text { in } D, \quad w \mid \partial D=0
$$

and.

$$
\Delta w+\delta \lambda_{N_{+}+1} w=0 \quad \text { in } D, \quad w \mid \partial D=0
$$

9 - Annali di Matematica 
If $\left\{y_{k}\right\}_{1}^{\infty}$ and $\left\{\delta_{k}\right\}_{1}^{\infty}$ denote the eigenvalues of (37) and (38) respectively, indexed in order of increasing magnitude, then clearly

$$
\gamma_{k}=\lambda_{k} / \lambda_{N+1}, \quad \delta_{k}=\lambda_{k} / \lambda_{N+1}
$$

From the condition $\operatorname{tg}^{\prime \prime}(t)>0$ a.e. and conditions $(a),(b)$, and $(c)$ of Theorem $B$ we have

$$
\lambda_{N-1}<g^{\prime}(0) \leqslant g^{\prime}(t) \leqslant \max \left\{g^{\prime}(\infty), g^{\prime}(-\infty)\right\}<\lambda_{N+1}
$$

for all $t \in(-\infty, \infty)$. Hence

$$
\lambda_{N-1}<\int_{0}^{1} g^{\prime}\left(s u_{0}(x)\right) d s \leqslant g^{\prime}\left(u_{0}(x)\right)<\lambda_{N+1}
$$

for all $x \in D$. From (39) and the same type of comparison argument that led to (36) we have the inequalities

$$
\begin{aligned}
& \alpha_{N-1}<\delta_{N-1}=1, \\
& 1=\gamma_{N+1}<\alpha_{N+1}, \\
& 1=\gamma_{N+1}<\beta_{N+1} .
\end{aligned}
$$

Since $u_{0}(x) \neq 0$, it follows from (30) that 1 is eigenvalue of $(32)$; hence from (40) and (41) we see that $\alpha_{N}=1$. The claim (34) now follows from (42) and (36) with $k=N$.

If

$$
V=\operatorname{span}\left\{\theta_{1}, \ldots, \theta_{N}\right\}
$$

then a straight forward calculation shows that

$$
\langle v, v\rangle_{1} \leqslant \beta_{R} \int_{0} g^{\prime}\left(u_{0}(x)\right) v^{2} d x \quad \text { if } v \in V .
$$

(Compare with (20)). Therefore, from (23), we conclude that

$$
\left\langle D^{2} F\left(u_{0}\right) v, v\right\rangle_{1} \leqslant\left(1-\frac{1}{\beta_{N}}\right)\langle v, v\rangle_{1} \quad \text { if } v \in V .
$$

To prove the first assertion of Lemma 3 we show that all of the eigenvalues of the self-adjoint operator $D^{2} G\left(x_{0}\right): X \rightarrow X$ are negative. Assuming the contrary, there exists $h_{1} \in X$ such that $\left\langle D^{2} G\left(x_{0}\right) h_{1}, h_{1}\right\rangle_{1} \geqslant 0$. Let

$$
\bar{m}=h_{1}+\varphi^{\prime}\left(x_{0}\right)\left(h_{1}\right) \text {. }
$$


From (12) we have

$$
\left\langle D^{2} F\left(u_{0}\right) \bar{m}, \bar{m}\right\rangle_{1} \geqslant 0,
$$

and from (11) we see that

$$
\left\langle D^{2} F\left(u_{0}\right) \bar{m}, k\right\rangle_{1}=0 \quad \text { if } k \in Y \text {. }
$$

Recall, from Lemma 2, that $\left\langle D^{2} F\left(u_{0}\right) k, k\right\rangle_{1} \geqslant m\|k\|_{1}^{2}$ with $m>0$ if $k \in Y$. Therefore, by (45), (46), and the self-adjointness of $D^{2} F\left(u_{0}\right)$, if $k \in Y$ and $\alpha \in R$, then

$$
\left\langle D^{2} F\left(u_{0}\right)(k+\alpha \bar{m}), k+\alpha \bar{m}\right\rangle_{1}=\left\langle D^{2} F\left(u_{0}\right) k, k\right\rangle_{1}+\alpha^{2}\left\langle D^{2} F\left(u_{0}\right) \bar{m}, \bar{m}\right\rangle_{1} \geqslant 0 .
$$

Thus, if $Z$ is the subspace of $H$ defined by

$$
Z=\{z \in H \mid z=k+\alpha \bar{m}, k \in Y, \alpha \in R\}
$$

then

$$
\left\langle D^{2} F\left(u_{0}\right) z, z\right\rangle_{1} \geqslant 0 \quad \text { if } z \in Z \text {. }
$$

Extend $h_{1}$ to a basis $\left\{h_{1}, \ldots, h_{N}\right\}$ of $X$ and let

$$
\hat{X}=\operatorname{span}\left\{h_{2}, \ldots, h_{N}\right\} .
$$

Since $H=X \oplus Y$, we infer from (45) and (48) that $H=\hat{X} \oplus Z$. Consequently,

$$
\theta_{k}=l_{k}+z_{k}, \quad l_{k} \in \hat{X}, \quad z_{k} \in Z, \quad 1 \leqslant k \leqslant N .
$$

Since $\operatorname{dim} \hat{X}=N-1$, there exist constants $c_{1}, \ldots, c_{N}$ not all zero such that $c_{1} l_{1}+$ $+\ldots+c_{N} l_{N}=0$. Therefore

$$
v \equiv c_{1} \theta_{1}+\ldots+c_{N} \theta_{N}=c_{1} z_{1}+\ldots+e_{N} z_{N} \in Z,
$$

and $v \neq 0$ by the independence of $\theta_{1}, \ldots, \theta_{N}$ : By $\left.(49)\left\langle D^{2} F\left(u_{0}\right) v, v\right\rangle_{1}\right\rangle 0$. On the other hand, from (34) and (44) it follows that $\left\langle D^{2} F\left(u_{0}\right) v, v\right\rangle_{1}<0$. This contradiction shows that all the eigenvalues of $D^{2} G\left(x_{0}\right)$ must be negative and the first assertion of Lemma 3 is proved.

The second assertion of Lemma 3 follows from the statement that $D^{2} G(0)$ has one positive eigenvalue and $N-1$ negative eigenvalues. This statement in turn is equivalent to the conditions: (i) $D^{2} G(0)$ is nonsingular; (ii) The quadratic form associated with $D^{2} G(0)$ cannot be negative definite on all of $X$; (iii) $D^{2} G(0)$ canno the positive definite on any two dimensional subspace of $X$. 
The first condition follows from Lemma 2 since, as shown in the proof of Theorem 4, condition (iii*) of Theorem 4 implies that 0 is a nondegenerate critical point of $G$.

To establish the second condition assume on the contrary that $\left\langle D^{2} G(0) h, h\right\rangle_{1}<0$ if $h \in X$ and $h \neq 0$. If $\hat{W}=\left\{h+\varphi^{\prime}(0)(h) \mid h \in X\right\}$ then $\operatorname{dim} \hat{W}=\operatorname{dim} X=N$ and, since $\phi(0)=0$, we infer from (12) that $\left\langle D^{2} F(0) w, w\right\rangle_{1}<0$ for $w \neq 0$ and $w \in \hat{W}$. Since the codimension of $Y_{1}$ is $N-1$, a standard algebraic argument, which has been used above, shows that there exists $w_{1} \in \hat{W} \cap Y_{1}$ with $w_{1} \neq 0$. Therefore $\left\langle D^{2} F(0) w_{1}\right.$, $\left.w_{1}\right\rangle_{1}<0$ and, from Lemma 2 and condition (iv) of Theorem $4,\left\langle D^{2} F(0) w_{1}, w_{1}\right\rangle_{1}$ $\geqslant m_{1}\left\|w_{1}\right\|_{1}^{2}>0$. This contradiction shows that $D^{2} G(0)$ cannot be negative definite on $X$.

To prove the third condition suppose that $Q$ is a two-dimensional subspace of $X$ such that $\left\langle D^{2} G(0) q, q\right\rangle_{\mathbf{1}}>0$ if $q \in Q$ and $q \neq 0$. If $\hat{Q}$ is the subspace of $H$ defined by

$$
\widehat{Q}=\left\{q+\varphi^{\prime}(0)(q) \mid q \in Q\right\}
$$

then according to (12), $\left\langle D^{2} F^{\prime}(0) \hat{q}, \hat{q}\right\rangle_{\mathbf{1}}>\mathbf{0}$ if $\hat{q} \neq 0$ and $\hat{q} \in \hat{Q}$. If $k \in Y$, by (11), $\left\langle D^{2} F(0) \hat{q}, k\right\rangle=0$. Thus, by condition (viii) of Theorem 4 , if $\hat{q} \in \hat{Q}$ and $k \in Y$, then $\left\langle D^{2} F(0)(\hat{q}+k), \hat{q}+k\right\rangle_{1}>0$ unless $\hat{q}=k=0$; from which we also infer that $\hat{Q} \cap Y=\{0\}$. Since codimension $(\hat{Q} \oplus Y)=$ codimension $Y$-dimension $\hat{Q}=N-2$ and $\operatorname{dim} X_{1}=N-1$ there exists $x_{1} \in X_{1} \cap(\hat{Q} \oplus Y)$ with $x_{1} \neq 0$. From Lemma 2, (iii*) of Theorem 4, and the above $\left\langle D^{2} F(0) x_{1}, x_{1}\right\rangle_{1}<0$ and $\left.\left\langle D^{2} F(0) x_{1}, x_{1}\right\rangle_{1}\right\rangle 0$. This contradiction proves that $D^{2} G\left(x_{0}\right)$ cannot have two positive eigenvalues, and by earlier remarks completes the proof of Lemma 3 .

As a by-product of the proof of Lemma 3 , we have the result that if $u_{0}$ is any solution of $\left(P_{0}\right)$ then the boundary value problem

$$
\Delta w+g^{\prime}\left(u_{\theta}(x)\right) w=0 \quad \text { in } D \quad w \mid \partial D=0
$$

(in the generalized sense), has only the trivial solution assuming the conditions of Theorem $B$. This follows from (33) and (34) if $u_{0}$ is not identically zero and is a trivial consequence of condition $(a)$ otherwise. By using the same argument, based on the inverse function theorem, that was used in the proof of the second assertion of Theorem $A$ we have

LEMMA 4. - If $u_{0}$ is a solution of $\left(P_{0}\right)$, then, under the conditions of Theorem $B$, there exist numbers $\delta>0$ and $\delta^{\prime}>0$ such that if $p \in L^{2}(D)$ and $|p|_{0}<\delta$, then there exists a unique weak solution $u$ of $(P)$ with $\left|u-u_{0}\right|_{1}<\delta^{\prime}$.

LEMMA 5. - Given $r>0$ there exists a number $R(r)>0$ such that if the conditions of Theorem $B$ hold and $p \in L^{2}(D)$ with $|p|_{0} \leqslant r$ then any weak solution of $(P)$ satisfies $|u|_{1} \leqslant R(r)$. 
Proof. - If $\gamma$ and $\gamma^{\prime}$ are numbers such that

$$
\lambda_{N}<\gamma^{\prime} \leqslant \max \left\{g^{\prime}(\infty), g^{\prime}(-\infty)\right\} \leqslant \gamma<\lambda_{N+1}
$$

then according to conditions $(b)$ and $(c)$ of Theorem $B$ there exists a number $t_{0}>0$ such that $\gamma^{\prime} \leqslant g(t) / t \leqslant \gamma$ if $|t| \geqslant t_{0}$. We can extend the restriction of $g(t) / t$ to $(-\infty$, $\left.-t_{0}\right] \cup\left[t_{0}, \infty\right.$ ) (for example, linearly between $-t_{0}$ and $t_{0}$ ) to a function $h(t)$ continuous on $(-\infty, \infty)$ with

$$
\lambda_{N}<\gamma^{\prime} \leqslant h(t) \leqslant \gamma<\lambda_{N+1}
$$

for all $t$. Since the function $g(t)-h(t) t$ is continuous and has compact support, it is bounded. Hence

$$
g(t)=h(t) t+H(t), \quad|H(t)| \leqslant L
$$

for some constant $L$. Suppose $p \in L^{2}(D)$ and $|p|_{0} \leqslant r$. Let $u$ be a weak solution of $(P)$. If $w \in \stackrel{\circ}{H}_{1}(D)$ then

$$
\int_{D}(\langle\nabla u, \nabla w\rangle-g(u) w+p(\xi) w) d \xi=0
$$

Let $u=x+y$ with $x \in X$ and $y \in Y$ and choose $w=y-x$. Since $Y=X^{\perp},|w|_{1}=$ $=|u|_{1}$. From (52) we have

$$
\langle y-x, y+x\rangle_{1}-\int_{D} h(u)\left(y^{2}-x^{2}\right) d \xi=\int_{D}(H(u(\xi)) w(\xi)-p(\xi) w(\xi)) d \xi
$$

Hence, by (51),

$$
|y|_{1}^{2}-\gamma|y|_{0}^{2}+\gamma^{\prime}|x|_{0}^{2}-|x|_{1}^{2} \leqslant\left(L(\operatorname{meas} D)^{\frac{1}{2}}+|p|_{0}\right)|w|_{0} \leqslant\left(L(\operatorname{meas} D)^{\frac{1}{2}}+r\right)\left(1 / \sqrt{\lambda_{1}}\right)|w|_{1} .
$$

Therefore, using (19) and (20) we have

$$
\left(1-\frac{\gamma}{\lambda_{N+1}}\right)|y|_{0}^{2}+\left(\frac{\gamma^{\prime}}{\lambda_{N}}-1\right)|x|_{1}^{2} \leqslant\left(L(\operatorname{meas} D)^{\frac{1}{2}}+r\right)\left(1 / \sqrt{\lambda_{1}}\right)|u|_{1} .
$$

Thetting $b>0$ denote the minimum of the two numbers $\left(1-\gamma / \lambda_{N-1}\right)$ and $\left(\gamma^{\prime} / \lambda_{N}-1\right)$ we see that

$$
|u|_{1} \leqslant\left(L(\text { meas } D)^{\frac{1}{2}}+r\right)\left(1 / b \sqrt{\lambda_{1}}\right) \equiv R(r)
$$

and the lemma is proved.

Lenna 6. - Under the conditions of Theorem $B$ there exist exactly three solutions -one trivial and two nontrivial-of the homogeneous problem $\left(P_{0}\right)$. 
Proof. - By Lemma 1, it is sufficient to show that there are exactly three solutions of $\nabla G(x)=0$. If $\nabla G(x)=0$ then $u=x+\varphi(x)$ is a solution of $\nabla F(u)=0$ or, equivalently, $u$ is a weak solution of $\left(P_{0}\right)$. Hence, by Lemma $5,|u|_{1} \leqslant R(0)$. Since $\varphi(x) \in$ $\in Y=X^{\perp}$, it follows that $|x|_{1} \leqslant R(0)$. Now, according to Lemma 3 and the inverse function theorem, the solutions of $\nabla G(x)=0$ are isolated. Hence, there can exist only a finite number of solutions of $\nabla G(x)=0$. Let $x_{1}, \ldots, x_{k}$ denote the nonzero solutions of $\nabla G(x)=0$; by Theorem $A, k \geqslant 2$. The critical points of $G$ and $f=-G$ coincide, so according to Lemma 3 sgn det $D^{2} f(0)=(-1)^{N} \operatorname{sgn} \operatorname{det} D^{2} G(0)=-1$, and, if $1 \leqslant i \leqslant k$, sgn det $D^{2} f\left(x_{i}\right)=(-1)^{N} \operatorname{sgn} \operatorname{det} D^{2} G\left(x_{i}\right)=1$. By (13), $f(x) \rightarrow \infty$ as $|x|_{1} \rightarrow \infty$ so $f$ satisfies the conditions of Theorem 2 . Since $i\left(\nabla f, x_{j}\right)=\operatorname{sgn} \operatorname{det} D^{2} f\left(x_{j}\right)$, it follows from Theorem 2 and the above that $-1+k=1$. Hence $k=2$ and the Lemma is proved.

To complete the proof of Theorem $B$ let $u_{0}, u_{1}$, and $u_{2}$ be the three solutions of the problem $\left(P_{0}\right)$. According to Lemma 4 , we can choose numbers $\delta>0$ and $\delta^{\prime}>0$ such that if $p \in L^{2}(D)$ and $|p|_{0}<\delta$ then there exist solutions $\tilde{u}_{k}, k=0,1,2$ of problem $(P)$ with $\left|\tilde{u}_{k}-u_{k}\right|_{1}<\delta^{\prime}$ for $k=0,1,2$. Since $\delta$ and $\delta^{\prime}$ can be taken to be arbitrarily small, these solutions will be distinct if $|p|_{0}$ is small.

Assuming that Theorem $B$ is not true, there exists a sequence $\left\{p_{m}\right\}_{1}^{\infty}$ in $L^{2}(D)$ such that $\left|p_{m}\right|_{0} \rightarrow 0$ as $m \rightarrow \infty$ and such that there exist four distinct solutions $u_{l m}$, $l=0,1,2,3$, of $(P)$ with $p=p_{m}$. Consequently, if $w \in \stackrel{H}{1}_{1}(D)$ and $0 \leqslant l \leqslant 3$, then

$$
\int_{D}\left(\left\langle\nabla w, \nabla u_{l m}\right\rangle-g\left(u_{l_{m}}\right) w+w p_{m}\right) d \xi=0
$$

If $N: \stackrel{\circ}{H}_{1}(D) \rightarrow \stackrel{\circ}{H}_{1}(D)$ is defined by $\langle N(u), w\rangle_{1}=\int_{D} g(u) w d \xi$ then since $g$ has a bounded derivative and the injection $\stackrel{\circ}{H}_{1}(D) \rightarrow L_{2}(D)$ is compact ([4]), $N$ is continuous with respect to weak convergence. From the Riesz representation theorem there exists $v_{m} \in \stackrel{\circ}{H}_{1}(D)$ such that $\left\langle w, p_{m}\right\rangle_{0}=\left\langle w, v_{m}\right\rangle_{1}$ and, since

$$
\left|v_{m}\right|_{1}^{2} \leqslant\left|p_{m}\right|_{0}\left|v_{m}\right|_{0} \leqslant\left|p_{m}\right|_{0}\left(1 / \sqrt{\lambda_{1}}\right)\left|v_{m}\right|_{1}, \quad\left|v_{m}\right|_{1} \rightarrow 0 \text { as } m \rightarrow \infty
$$

We can thus write (53) in the form

$$
\left\langle u_{l m}, w\right\rangle_{1}=\left\langle N\left(u_{l_{m}}\right), w\right\rangle_{1}-\left\langle v_{m}, w\right\rangle_{1}
$$

for all $w \in \stackrel{ }{H}_{1}(D)$; hence

$$
u_{l m}=N\left(u_{l m}\right)-v_{m}, \quad 0 \leqslant l \leqslant 3
$$

Since the sequence $\left\{p_{m}\right\}_{1}^{\infty}$ is bounded in $L^{2}(D)$, it follows from Lemma 5 that the sequences $\left\{u_{t_{m}}\right\}_{m=1}^{\infty}$ are bounded in $\mathscr{H}_{1}(D)$. Hence, there is a sequence of integers $\left\{m_{j}\right\}_{j=1}^{\infty}$ such that $\left\{u_{l_{m_{t}}}\right\}_{j=1}^{\infty}$ converges weakly to some $z_{l}$ in $\stackrel{\circ}{H}_{1}(D)$ for $0 \leqslant l \leqslant 3$. Since $N\left(u_{l m_{\mathrm{j}}}\right)$ converges strongly to $N\left(z_{l}\right)$ for $l=0,1,2,3$, it follows from $(54)$ that 
$\left\{u_{l m}\right\}_{1}^{\infty}$ actually converges strongly to $z_{l}$ and $z_{l}=N\left(z_{l}\right)$ for $0 \leqslant l \leqslant 3$. Hence, for $w \in \ddot{H}_{1}(D),\left\langle z_{l}, w\right\rangle_{1}=\left\langle N\left(z_{l}\right), w\right\rangle_{1}$ or $\int_{D}\left(\left\langle\nabla z_{l}, w\right\rangle-g\left(z_{l}\right) w\right) d \xi=0$ if $l=0,1,2,3$, so $z_{l}$ is a weak solution of $\left(P_{0}\right)$. Therefore, each $z_{l}, l=0,1,2,3$, is equal to some $u_{k}$, $k=0,1,2$. This means that some two of the four sequences $\left\{u_{l u}\right\}_{j=1}^{\infty}, 0 \leqslant l \leqslant 3$ must converge to the same weak solution of $\left(P_{\mathbf{0}}\right)$. Since for each $j$, the four functions $u_{l m_{3}}$, $l=0,1,2,3$ are distinct, and since $\left|p_{m_{j}}\right|_{0} \rightarrow 0$ as $j \rightarrow \infty$, this contradicts Lemma 4 for $j$ large and Theorem $B$ is proved.

\section{5. - Proof of Theorem $C$.}

To prove Theorem $O$ we make use of the following result due to CLARK $[8$, p. 71] which was actually stated in the more general context of $O^{1}$ functions defined on Banach spaces which have a second derivative only at the origin:

Let $H$ be a real Hilbert space and $f$ an even, real-valued $C^{2}$ function defined on $H$. Suppose that $f$ has the property that whenever $\left\{x_{n}\right\} \subseteq H$ is a bounded sequence such that $f\left(x_{n}\right)<0, f\left(x_{n}\right)$ is bounded below, and $\nabla f\left(x_{n}\right) \rightarrow 0$, then $\left\{x_{n}\right\}$ contains a convergent subsequence. Suppose that $f(0)=0, f$ is bounded betow, there exists a subspace $M$ of $H$ of dimension $l>0$ such that $\left\langle D^{2} f(0) x, x\right\rangle<0$ if $x \in M$ with $x \neq 0$, and $f(x) \geqslant 0$ for $|x|$ sufficiently large. Then there exist at last $2 l$ nonzero solutions of $\nabla f(x)=0$.

To prove Theorem $O$ we again define $F: \stackrel{\circ}{H}_{1}(D) \rightarrow R$ by (22) and observe that, since $g$ is odd, $F$ is even. As shown in the proof of Theorem $A, F$ satisfies the hypotheses of Theorem 4 . We assert that the function $\varphi$ defined by $(7)$ and (8) is odd. Indeed, by the oddness of $\nabla F$, it follows from (7) that $\langle\nabla F(-x-\varphi(x)), k\rangle=$ $=-\langle\nabla F(x+\varphi(x)), k\rangle=0$ for all $k \in Y$. Since $\varphi(-x)$ is the unique element of $Y$ such that $\langle\nabla F(-x+\varphi(-x)), k\rangle=0$ for all $k \in Y$, it follows that $\varphi(-x)=-\varphi(x)$. From this it follows that the function $G: X \rightarrow R$ defined by $G(x)=F(x+\varphi(x))$ is also even.

We assert that, under the conditions of Theorem $C$, the quadratic form associated with $D^{2} G(0)$ is positive definite on some subspace $M$ of $X$ of dimension $N-K+1$. Assuming the contrary, $D^{2} G(0)$ has at least $K$ nonpositive eigenvalues so there exists a subspace $W$ of $X$ with $\operatorname{dim} W \geqslant K$ such that $\left\langle D^{2} G(0) w, w\right\rangle \leqslant 0$ for all $w \in W$. If $\hat{W}$ is the subspace of $\stackrel{\circ}{H}_{1}(D)$ defined by $\hat{W}=\left\{w+\varphi^{\prime}(0) w \mid w \in W\right\}$, then, since $\varphi(0)=0$, it follows from (12) that $\left\langle D^{2} F(0) v, v\right\rangle \leqslant 0$ for all $v \in \hat{W}$. By (21) and the hypotheses of Theorem $C$, it follows that if $Y_{1}$ is defined as in the proof of Theorem $A$, then there exists $m_{1}>0$ such that $\left\langle D^{2} F(0) s, s\right\rangle \geqslant m_{1}\langle s, s\rangle$ for all $s \in Y_{1}$ and codimension $Y_{1}=K-1$. Since $\operatorname{dim} \hat{W}=\operatorname{dim} W \geqslant K$ there exists $z \in \hat{W} \cap Y_{1}$ with $z \neq 0$ which is clearly impossible. This contradiction establishes the existence of an $(N-K+1)$-dimensional subspace $M$ of $X$ on which $D^{2} G(0)$ is positive definite. 
If $f(x)=-G(x)$ then $\left\langle D^{2} f(0) x, x\right\rangle<0$ if $x \in M$ and $x \neq 0$. Moreover, by (13), $f(x) \rightarrow+\infty$ as $|x| \rightarrow \infty$. Since $\operatorname{dim} X=N<\infty$, it is clear that $f: X \rightarrow R$ satisfies all of the conditions of the aforementioned result due to Clark. This establishes the existence of at least $2(N-K+1)$ nonzero solutions of $\nabla G(x)=0$, and so by Lemma 1 of the previous section, there exist at least $2(N-K+1)$ nonzero solutions of $\nabla F(u)=0$. Since critical points of $F$ are weak solutions of $\left(P_{0}\right)$ this proves Theorem $O$.

\section{6. - Regularity.}

The fact that, under the hypotheses of Theorem $A$, any weak $\stackrel{\circ}{H}_{1}$-solution of $(P)$ is also a classical solution follows from a standard "bootstrap » argument which we indicate for completeness.

Let $W_{m, p}(D)$ denote the standard Sobolev space of functions having generalized $L^{p}(D)$-derivatives up to order $m \geqslant 1$. If $u$ is a weak solution of $(P)$ in $\stackrel{\circ}{H}_{1}(D) \subset W_{1,2}$ then $u \in L^{2}(D)$. Assume that we have established that $u \in L^{\alpha}(D)$ for some $q \geqslant 2$. Since $g^{\prime}$ is bounded and $p(x) \in C^{\alpha}(D)$, for some $\alpha \in(0,1)$,

$$
h(x) \equiv p(x)-g(u(x)) \in L^{a}(D) .
$$

Therefore, since $u$ is a generalized solution of

$$
\begin{aligned}
(\Delta u)(x) & =h(x), & & x \in D \\
u(x) & =0, & & x \in \partial D,
\end{aligned}
$$

if follows from a result due to AgMon, Douglis and Nirenberg [1] that $u \in W_{2, q}$ : If $2 q \geqslant n$ then by the Sobolev imbedding theorem (see [4, p. 221]) it follows that $u \in L^{r}(D)$ for any $r \in[1, \infty)$. If $2 q<n$ then $u \in L^{r}(D)$ where $r=n q /(n-2 q)>q$. Repeating this argument a sufficient number of times, we can conclude that $u \in W_{2, r}$ where $r$ is so large that $0<\alpha<1-(n / r)$. Since $W_{2, r} \subseteq W_{1, r}$, it follows from a result due to Morrey [19, Theorem 3.3.3] that $u \in C^{\beta}(D)$ where the Hölder exponent $\beta=1-(n / r)$. Since $g^{\prime}$ is continuous and $C^{\beta}(D) \subseteq O^{\alpha}(D), h(x)=p(x)-g(u(x)) \in C^{\alpha}(D)$. Therefore, since $\partial D$ is of class $C^{2+\alpha}$, it follows by standard potential theory [10] that there exists a solution of

$$
\begin{aligned}
\Delta(v)(x) & =h(x), & & x \in D \\
v(x) & =0, & & x \in \partial D,
\end{aligned}
$$

with $v \in C^{2+\alpha}$. Since $u$ and $v$ are both $\stackrel{\circ}{H}_{1}$-weak solutions of (53) and (55) has a unique $\stackrel{\circ}{H}_{1}$-weak solution, $u=v$. This proves that $u$ is a classical solution of $(P)$. 


\section{REFERENCES}

[1] S. Agmon - A. Dovglis - L. NIRmnberg, Estimates near the boundary for solutions of elliptie partial differential equations satisfying general boundary conditions, $I$, Comm. Pure Appl. Math., 42 (1959), pp. 623-727.

[2] A. Ambrosetri - G. Prodr, Ont he inversion of some differentiable mappings with singularities between Banach spaces, Annali Mat. Pura Appl., 93 (1972), pp. 231-246.

[3] S. BANCROFT - J. K. HAXE - D. SwEET, Alternative problems and nonlinear functional equations, J. Diff. Egns., 4 (1968), pp. 40-56.

[4] L. Bers - F. JoHn - M. SChEchter, Partial differential equations, Interscience, New York, 1964.

[5] A. Castro - A. Lazer, Applications of a max-min principle, Rev. Colombiana Mat., 4h (1976), pp. 141-149.

[6] L. CeSARI, Functional analysis and Gelerkin's method, Mich. Math. J., 44 (1964), pp. $385-418$.

[7] N. Chow - J. HaLe - J. Marcert-PareT, Applications of generie bifureation, I, Arch. Rat. Mech. Anal., 2 (1975), pp. 159.188.

[8] D. CLARK, A variant of the Lusternik-Schnirelman theory, Indiana Univ. Math. J., 22 (1972), pp. 65-74.

[9] R. Covrant - D. Hubert, Methods of mathematical physics, vol. I, Interscience, New York, 1953.

[10] R. Courant - D. Hilbert, Methods of mathematical physics, vol. 2, Interscience, New York, 1962.

[11] C. DoLPH, Nonlinear integral equations of the Hammerstein type, Trans. Amer. Math. Soc., 66 (1949), pp. $289-307$.

[12] M. Greenberg, Lectures on algebraic topology, W. A. Benjamin, Inc., Reading, Mass., 1967.

[13] J. KAZDAN - F. WARarer, Remarks on some quasilinear elliptic equations, Comm. Pure Appl. Math., 28 (1975), pp. 567-597.

[14] E. LANDESMAN - A. LAZER, Linear eigenvalues and a nonlinear boundary value problem, Pacific J. of Math., 33 (1970), pp. 311-328.

[15] S. LANG, Differential Manifolds, Addison-Wesley, Reading, Mass., 1972.

[16] A. Lazer - E. Landesman - D. Meyers, On saddle point problems in the caleulus of variations, the Ritz algorithm, and monotone convergence, J. Math. Anal. Appl., 53 (1975), pp. 594-614.

[17] J. MrLnor, Topology from the differentiable viewpoint, University Press of Virginia, Charlottesville, 1965.

[18] C. Mrranda, Partial differential equations of elliptic type, Springer-Verlag, New York, Heidelberg, Berlin, 1970.

[19] C. Mornex, Multiple integrals in the calculus of variations, Springer, New York, 1966.

[20] P. Rabinowitz, A note on topological degree for potential operators, J. Math. Anal. Appl., 51. (1975), pp. 483-492.

[21] E. Rothe, A relation between the type numbers of a oritical point and the index of the corresponding field of gradient vectors, Math. Nacht., 4 (1950-51), pp. 12-27.

[22] E. Spanier, Algebraic topology, McGraw-Hill, New York, 1966.

[23] M. VAINBerg, Variational Methods for the Study of Nonlinear Operators, Holden-Day, San Francisco, 1964. 